\title{
Vertex Connected Domination Polynomial of some Coalescence of Complete and Wheel Graphs
}

\author{
Nechirvan Badal Ibrahim \\ Department of Mathematics, College of Science, University of Duhok, Kurdistan Region, Iraq \\ Corresponding Author: nechirvan.badal@uod.ac

\section{Hariwan Fadhil M.Salih} \\ Department of Mathematics, College of Science, University of Duhok, Kurdistan Region, Iraq
}

Received: 21 September 2020; Accepted: 18 November 2020; Published: 08 December 2020

\begin{abstract}
In this paper, we introduce new results of vertex connected dominating set and vertex connected domination polynomial of vertex identification, edge introduced and t-tuple of complete graph, also we determine new results of vertex connected dominating set and vertex connected domination polynomial of vertex identification, edge introduced and t-tuple of wheel graph .
\end{abstract}

Index Terms: Coalescence graphs, Vertex connected dominating set, Vertex connected domination polynomial.

\section{Introduction}

A vertex connected dominating set $F$ is a set of vertices of a graph $G=(V, E)$ such that every vertex in $V-F$ is adjacent to at least one vertex in $\mathrm{F}$ and the sub-graph $<\mathrm{F}>$ induced by the set $\mathrm{F}$ is connected. The vertex connected domination number $\gamma_{v c}(G)$ is the minimum of the cardinalities of the vertex connected dominating sets of $\mathrm{G}$.

The domination polynomial was first introduced by the author in his Ph.D. thesis in [12]. The connected domination number of a graph was studied in [5]. And the connected dominating set and connected domination polynomial was introduced in [9]. For more information and motivation of domination polynomial and connected domination polynomial refer to $[1,2,3,4,7,10,11,13,14,15]$.

A simple graph in which each pair of distinct vertices is joined by an edge is called a complete graph, which is denoted by $K_{n}$, and the wheel graph is the join of $\mathrm{K} 1$ and cycle graph, that means $W_{n}=K_{1}+C_{n-1}$. The vertex identification graph $G_{1} \bullet G_{2}$ is obtained from G1 and $\mathrm{G} 2$ by identifying the vertex $\mathrm{u} \in \mathrm{G} 1$ with $\mathrm{v} \in \mathrm{G} 2$. The edge introduced graph $G_{1}: G_{2}$ of two graphs is obtained from $\mathrm{G} 1$ and $\mathrm{G} 2$ by introducing the edge e=uv between a vertex $\mathrm{u} \in \mathrm{G} 1$ with a vertex $\mathrm{v} \in \mathrm{G} 2[6,8]$.

Let $\left\{\left(\mathrm{G}_{1}, \mathrm{u}_{1}\right),\left(\mathrm{G}_{2}, \mathrm{u}_{2}\right), \ldots,\left(\mathrm{G}_{\mathrm{t}}, \mathrm{u}_{\mathrm{t}}\right)\right\}$ be a family of not necessary distinct connected graphs with roots $\mathrm{u}_{1}, \mathrm{u}_{2}, \ldots, \mathrm{u}_{\mathrm{t}}$, respectively. A connected graph $\mathrm{G}=\mathrm{G}_{1} \circ \mathrm{G}_{2} \circ \ldots \circ \mathrm{G}_{\mathrm{t}}$ is called the multiple coalescence of $\mathrm{G}_{1}, \mathrm{G}_{2}, \ldots, \mathrm{G}_{\mathrm{t}}$ provided that the vertices $\mathrm{u}_{1}, \mathrm{u}_{2}, \ldots, \mathrm{u}_{\mathrm{t}}$ are identified to reform the coalescence vertex $\mathrm{u}$. The t-tuple coalescence graph is denoted by $G^{|t|}$ is the multiple coalescence of $t$ isomorphic copies of a graph $G$ [8].

In this paper we determine new results of vertex connected domination polynomial of vertex identified graph; edge introduced graph and t-tuple coalescence graph of some standard graphs, for instance, complete and wheel graphs.

Definition 1.1:[9] Let $\mathrm{G}$ be a connected graph of order $\mathrm{n}$ and let $d_{v c}(G, i)$ denoted the number of vertex connected dominated sets with cardinality $i$. Then the vertex connected domination polynomial $D_{v c}(G, x)$ of $\mathrm{G}$ is defined by

$$
D_{v c}(G, x)=\sum_{i=\gamma_{v c}(G)}^{n} d_{v c}(G, i) x^{i}
$$

where $\gamma_{v c}(G)$ is the vertex connected domination number of $\mathrm{G}$ and the roots of the vertex connected domination polynomial of $\mathrm{G}$ is denoted by $Z\left(D_{v c}(G, x)\right)$. 


\section{Theorem 1.2: [9]}

1) For any complete graph $K_{n}$ of n vertices, then,

$$
D_{v c}\left(K_{n}, x\right)=(1+x)^{n-1} .
$$

2) For any wheel graph $W_{n}$ with order $\mathrm{n}$. Then,

$$
D_{v c}\left(W_{n}, x\right)=x(1+x)^{n-1}+x^{n+1}+(n-1) x^{n-2}+(n-1) x^{n-3} .
$$

\section{Vertex Connected Domination Polynomial of complete Coalescence Graphs}

In this section, we introduce new results of vertex connected dominating set and vertex connected domination polynomial of some coalescence graphs of complete $K_{n}$ graph.

Definition 2.1: Let $K_{n_{1}} \bullet K_{n_{2}}$ be the vertex identification graph of order $\mathrm{n}_{1}+\mathrm{n}_{2}-1$ and let $d_{v c}\left(K_{n_{1}} \bullet K_{n_{2}}, i\right)$ denoted the number of vertex connected dominating sets with cardinality i. Then the vertex connected domination polynomial of $K_{n_{1}} \bullet K_{n_{2}}$ is defined by

$$
D_{v c}\left(K_{n_{1}} \bullet K_{n_{2}}, x\right)=\sum_{i=\gamma_{v c}\left(K_{n_{1}} \bullet K_{n_{2}}\right)}^{n_{1}+n_{2}-1} d_{v c}\left(K_{n_{1}} \bullet K_{n_{2}}, i\right) x^{i},
$$

where $\gamma_{v c}\left(K_{n_{1}} \bullet K_{n_{2}}\right)$ is the vertex connected domination number of $K_{n_{1}} \bullet K_{n_{2}}$ and the roots of the vertex connected domination polynomial of $K_{n_{1}} \bullet K_{n_{2}}$ is denoted by $Z\left(D_{v c}\left(K_{n_{1}} \bullet K_{n_{2}}, x\right)\right)$.

Example 2.2: Consider the graph $K_{3} \bullet K_{3}$ as shown in Fig.1.

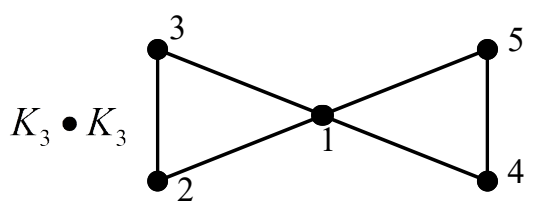

Fig.1. A graph $K_{3} \bullet K_{3}$.

We have:

If $\mathrm{i}=1, d_{v c}\left(K_{3} \bullet K_{3}, 1\right)=|\{1\}|=1$,

If $\mathrm{i}=2, d_{v c}\left(K_{3} \bullet K_{3}, 2\right)=|\{(1,2),(1,3),(1,4),(1,5)\}|=4$,

If $\mathrm{i}=3, d_{v c}\left(K_{3} \bullet K_{3}, 3\right)=|\{(1,2,3),(1,2,4),(1,2,5),(1,3,4),(1,3,5),(1,4,5)\}|=6$,

If $\mathrm{i}=4, d_{v c}\left(K_{3} \bullet K_{3}, 4\right)=|\{(1,2,3,4),(1,2,3,5),(1,2,4,5),(1,3,4,5)\}|=4$,

If $\mathrm{i}=5, d_{v c}\left(K_{3} \bullet K_{3}, 5\right)=|\{(1,2,3,4,5)\}|=1$,

Hence,

$D_{v c}\left(K_{3} \bullet K_{3}, x\right)=x^{5}+4 x^{4}+6 x^{3}+4 x^{2}+x$.

Proposition 2.3: The following properties hold for the coefficients of $D_{v c}\left(K_{n_{1}} \bullet K_{n_{2}}, x\right)$, for all $\mathrm{n}_{1}, \mathrm{n}_{2} \geq 3$.

i) $\quad d_{v c}\left(K_{n_{1}} \bullet K_{n_{2}}, 0\right)=0$.

ii) $\quad d_{v c}\left(K_{n_{1}} \bullet K_{n_{2}}, 1\right)=d_{v c}\left(K_{n_{1}} \bullet K_{n_{2}}, n_{1}+n_{2}-i\right)=1$.

iii) $d_{v c}\left(K_{n_{1}} \bullet K_{n_{2}}, 2\right)=d_{v c}\left(K_{n_{1}} \bullet K_{n_{2}}, n_{1}+n_{2}-2\right)=\left(\begin{array}{c}n_{1}+n_{2}-2 \\ 1\end{array}\right)$.

iv) $d_{v c}\left(K_{n_{1}} \bullet K_{n_{2}}, 3\right)=d_{v c}\left(K_{n_{1}} \bullet K_{n_{2}}, n_{1}+n_{2}-3\right)=\left(\begin{array}{c}n_{1}+n_{2}-2 \\ 2\end{array}\right)$.

v) $d_{v c}\left(K_{n_{1}} \bullet K_{n_{2}}, 4\right)=d_{v c}\left(K_{n_{1}} \bullet K_{n_{2}}, n_{1}+n_{2}-4\right)=\left(\begin{array}{c}n_{1}+n_{2}-2 \\ 3\end{array}\right)$.

vi) $d_{v c}\left(K_{n} \bullet K_{n}, i\right)=d_{v c}\left(K_{n} \bullet K_{n}, n_{1}+n_{2}-i\right)=\left(\begin{array}{c}n_{1}+n_{2}-2 \\ i-1\end{array}\right)$ 
Theorem 2.4: The vertex connected dominating set of $K_{n_{1}} \bullet K_{n_{2}}$ is given by

$$
d_{v c}\left(K_{n_{1}} \bullet K_{n_{2}}, i\right)=\left(\begin{array}{c}
n_{1}+n_{2}-2 \\
i-1
\end{array}\right) \text {. }
$$

If $\mathrm{n}_{1}=\mathrm{n}_{2}$, then $d_{v c}\left(K_{n} \bullet K_{n}, i\right)=\left(\begin{array}{c}2 n-2 \\ i-1\end{array}\right), \mathrm{i}=1,2, \ldots, 2 \mathrm{n}-1$.

Moreover, $\gamma_{v c}\left(K_{n_{1}} \bullet K_{n_{2}}, i\right)=1$.

Proof: By using Proposition 2.3, we get the result.

Theorem 2.5: The vertex connected domination polynomial of $K_{n_{1}} \bullet K_{n_{2}}$ is given by

$$
D_{v c}\left(K_{n_{1}} \bullet K_{n_{2}}, x\right)=x(x+1)^{n_{1}+n_{2}-2} .
$$

Proof: By using Definition 2.1 and Theorem 2.4, we have

$$
\begin{aligned}
D_{v c}\left(K_{n_{1}} \bullet K_{n_{2}}, x\right) & =\sum_{i=1}^{n_{1}+n_{2}-1} d_{v c}\left(K_{n_{1}} \bullet K_{n_{2}}, i\right) x^{i} \\
& =\sum_{i=1}^{n_{1}+n_{2}-1}\left(\begin{array}{c}
n_{1}+n_{2}-1 \\
i-1
\end{array}\right) x^{i} \\
& =\left(\begin{array}{c}
n_{1}+n_{2}-2 \\
0
\end{array}\right) x+\left(\begin{array}{c}
n_{1}+n_{2}-2 \\
1
\end{array}\right) x^{2}+\left(\begin{array}{c}
n_{1}+n_{2}-2 \\
2
\end{array}\right) x^{3}+\ldots+\left(\begin{array}{c}
n_{1}+n_{2}-2 \\
n_{1}+n_{2}-2
\end{array}\right) x^{n_{1}+n_{2}-1} \\
& =x\left[\begin{array}{c}
\left.1+\left(\begin{array}{c}
n_{1}+n_{2}-2 \\
1
\end{array}\right) x+\left(\begin{array}{c}
n_{1}+n_{2}-2 \\
2
\end{array}\right) x^{2}+\ldots+\left(\begin{array}{c}
n_{1}+n_{2}-2 \\
n_{1}+n_{2}-2
\end{array}\right) x^{n_{1}+n_{2}-2}\right] \\
\end{array}\right] \\
& =x\left[\begin{array}{c}
\sum_{i=0}^{n_{1}+n_{2}-2}\left(\begin{array}{c}
n_{1}+n_{2}-2 \\
i
\end{array}\right) x^{i} \\
i=
\end{array}\right. \\
& =x(x+1)^{n_{1}+n_{2}-1} .
\end{aligned}
$$

Hence, $D_{v c}\left(K_{n_{1}} \bullet K_{n_{2}}, x\right)=x(x+1)^{n_{1}+n_{2}-2}$.

And the vertex connected dominating roots are 0 with multiplicity 1 and -1 with multiplicity $n_{1}+n_{2}-2$.

Definition 2.6: Let $K_{n_{1}}: K_{n_{2}}$ be the edge introducing connected graphs of order $\mathrm{n}_{1}+\mathrm{n}_{2}$ and let $d_{v c}\left(K_{n_{1}}: K_{n_{2}}, i\right)$ denoted the number of vertex connected dominated sets with cardinality i. Then the vertex connected domination polynomial of $K_{n_{1}}: K_{n_{2}}$ is defined as

$$
D_{v c}\left(K_{n_{1}}: K_{n_{2}}, x\right)=\sum_{i=\gamma_{v c}\left(K_{n_{1}}: K_{n_{2}}\right)}^{n_{1}+n_{2}} d_{v c}\left(K_{n_{1}}: K_{n_{2}}, i\right) x^{i},
$$

where $\gamma_{v c}\left(K_{n_{1}}: K_{n_{2}}\right)$ is the vertex connected domination number of $K_{n_{1}}: K_{n_{2}}$.

Example 2.7: Consider the graph $\mathrm{K}_{4}: \mathrm{K}_{4}$ as shown in Fig. 2.

Fig. 2. A graph K4:K4.

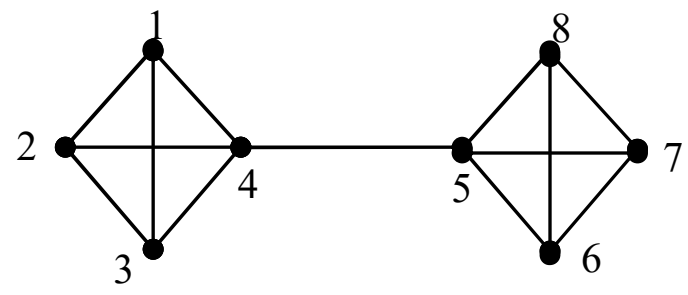

Then, we have:

If $\mathrm{i}=1, d_{v c}\left(K_{4}: K_{4}, 1\right)=0$,

If $\mathrm{i}=2, d_{v c}\left(K_{4}: K_{4}, 2\right)=|\{(4,5)\}|=1$,

If $\mathrm{i}=3, d_{v c}\left(K_{4}: K_{4}, 3\right)=|\{(1,4,5),(2,4,5),(3,4,5),(4,5,6),(4,5,7),(4,5,8)\}|=6$, 
If $\mathrm{i}=4, d_{v c}\left(K_{4}: K_{4}, 4\right)=\mid\{(1,2,4,5),(1,3,4,5),(1,4,5,6),(1,4,5,7),(1,4,5,8),(2,3,4,5),(2,4,5,6),(2,4,5$, $7),(2,4,5,8),(3,4,5,6),(3,4,5,7),(3,4,5,8),(4,5,6,7),(4,5,6,8),(4,5,7,8)\} \mid=15$,

If $\mathrm{i}=5, d_{v c}\left(K_{4}: K_{4}, 5\right)=\mid\{(1,2,3,4,5),(1,2,4,5,6),(1,2,4,5,7),(1,2,4,5,8),(1,3,4,5,6),(1,3,4,5,7),(1,3$, $4,5,8),(1,4,5,6,7),(1,4,5,6,8),(1,4,5,7,8),(2,3,4,5,6),(2,3,4,5,7),(2,3,4,5,8,(2,4,5,6,7),(2,4,5,6,8)$, $(2,4,5,7,8),(3,4,5,6,7),(3,4,5,6,8),(2,4,5,7,8),(4,5,6,7,8)\} \mid=20$,

If $\mathrm{i}=6, d_{v c}\left(K_{4}: K_{4}, 6\right)=\{(1,2,3,4,5,6),(1,2,3,4,5,7),(1,2,3,4,5,8),(1,3,4,5,6,7),(1,3,4,5,6,8),(1,2,4$, $5,6,7),(1,2,4,5,6,8),(1,2,4,5,7,8),(1,4,5,6,7,8),(2,3,4,5,6,7),(2,3,4,5,6,8),(1,3,4,5,7,8),(2,3,4,5$, $7,8),(2,4,5,6,7,8),(3,4,5,6,7,8)\} \mid=15$

If $\mathrm{i}=7, d_{v c}\left(K_{4}: K_{4}, 7\right)=6$,

If $\mathrm{i}=8, d_{v c}\left(K_{4}: K_{4}, 8\right)=1$,

Hence,

$D_{v c}\left(K_{4}: K_{4}, x\right)=x^{8}+6 x^{7}+15 x^{6}+20 x^{5}+15 x^{4}+6 x^{3}+x^{2}$.

Proposition 2.8: The following properties hold for the coefficients of $D_{v c}\left(K_{n_{1}}: K_{n_{2}}, x\right)$

$$
\begin{aligned}
& \text { i) } d_{v c}\left(K_{n_{1}}: K_{n_{2}}, 0\right)=d_{v c}\left(K_{n_{1}}: K_{n_{2}}, 1\right)=0 . \\
& \text { ii) } d_{v c}\left(K_{n_{1}}: K_{n_{2}}, 2\right)=d_{v c}\left(K_{n_{1}}: K_{n_{2}}, n_{1}+n_{2}\right)=\left(\begin{array}{c}
n_{1}+n_{2}-2 \\
0
\end{array}\right)=1 . \\
& \text { iii) } d_{v c}\left(K_{n_{1}}: K_{n_{2}}, 3\right)=d_{v c}\left(K_{n_{1}}: K_{n_{2}}, n_{1}+n_{2}-1\right)=\left(\begin{array}{c}
n_{1}+n_{2}-2 \\
1
\end{array}\right) . \\
& \text { iv) } d_{v c}\left(K_{n_{1}}: K_{n_{2}}, 4\right)=d_{v c}\left(K_{n_{1}}: K_{n_{2}}, n_{1}+n_{2}-2\right)=\left(\begin{array}{c}
n_{1}+n_{2}-2 \\
2
\end{array}\right) . \\
& \text { v) } d_{v c}\left(K_{n_{1}}: K_{n_{2}}, 5\right)=d_{v c}\left(K_{n_{1}}: K_{n_{2}}, n_{1}+n_{2}-3\right)=\left(\begin{array}{c}
n_{1}+n_{2}-2 \\
3
\end{array}\right) . \\
& \text { vi) } d_{v c}\left(K_{n_{1}}: K_{n_{2}}, i\right)=d_{v c}\left(K_{n_{1}}: K_{n_{2}}, n_{1}+n_{2}-(i-2)\right)=\left(\begin{array}{c}
n_{1}+n_{2}-2 \\
i-2
\end{array}\right) .
\end{aligned}
$$

Theorem 2.9: The vertex connected dominating set of edge introducing between two complete graphs is given by

$$
d_{v c}\left(K_{n_{1}}: K_{n_{2}}, i\right)=\left(\begin{array}{c}
n_{1}+n_{2}-2 \\
i-2
\end{array}\right) \text {, for all } 2 \leq i \leq n_{1}+n_{2} .
$$

If $n_{1}+n_{2}=n$, then $d_{v c}\left(K_{n}: K_{n}, i\right)=\left(\begin{array}{c}2 n-2 \\ i-2\end{array}\right), \mathrm{i}=2, \ldots, 2 \mathrm{n}$.

Moreover, $\gamma_{v c}\left(K_{n_{1}}: K_{n_{2}}\right)=2$.

Proof: By using Proposition 2.8, we get the result.

Theorem 2.10: The vertex connected domination polynomial of $K_{n_{1}}: K_{n_{2}}$ is given by

$$
D_{v c}\left(K_{n_{1}}: K_{n_{2}}, x\right)=x^{2}(x+1)^{n_{1}+n_{2}-2} .
$$

Proof: By using Definition 2.6, we have

$$
\begin{aligned}
D_{v c}\left(K_{n_{1}}: K_{n_{2}}, x\right) & =\sum_{i=2}^{n_{1}+n_{2}} d_{v c}\left(K_{n_{1}}: K_{n_{2}}, i\right) x^{i}, \text { and by Theorem 2.9, we have } \\
D_{v c}\left(K_{n_{1}}: K_{n_{2}}, x\right) & =\sum_{i=2}^{n_{1}+n_{2}}\left(\begin{array}{c}
n_{1}+n_{2}-2 \\
i-2
\end{array}\right) x^{i} \\
& =\left(\begin{array}{c}
n_{1}+n_{2}-2 \\
0
\end{array}\right) x^{2}+\left(\begin{array}{c}
n_{1}+n_{2}-2 \\
1
\end{array}\right) x^{3}+\left(\begin{array}{c}
n_{1}+n_{2}-2 \\
2
\end{array}\right) x^{4}+\ldots+\left(\begin{array}{l}
n_{1}+n_{2}-2 \\
n_{1}+n_{2}-2
\end{array}\right) x^{n_{1}+n_{2}} \\
& =x^{2}\left[\begin{array}{c}
\left.1+\left(\begin{array}{c}
n_{1}+n_{2}-2 \\
1
\end{array}\right) x+\left(\begin{array}{c}
n_{1}+n_{2}-2 \\
2
\end{array}\right) x^{2}+\ldots+\left(\begin{array}{l}
n_{1}+n_{2}-2 \\
n_{1}+n_{2}-2
\end{array}\right) x^{n_{1}+n_{2}-2}\right]
\end{array}\right.
\end{aligned}
$$




$$
\begin{aligned}
& =x^{2}\left[\sum_{i=0}^{n_{1}+n_{2}-2}\left(\begin{array}{c}
n_{1}+n_{2}-2 \\
i
\end{array}\right) x^{i}\right] \\
& =x^{2}(x+1)^{n_{1}+n_{2}-2} .
\end{aligned}
$$

Hence,

$$
D_{v c}\left(K_{n_{1}}: K_{n_{2}}, x\right)=x^{2}(x+1)^{n_{1}+n_{2}-2} .
$$

And the vertex connected dominating roots of $D_{v c}\left(K_{n_{1}}: K_{n_{2}}, x\right)$ are 0 with multiplicity 2 and -1 with multiplicity $n_{1}+n_{2}-2$.

Theorem 2.11: The vertex connected dominating set of t-tuple coalescence of complete graph $K_{n}$ is given by

$$
d_{v c}\left(K_{n}^{|t|}, i\right)=\left(\begin{array}{c}
t(n-1) \\
i-1
\end{array}\right) . \text { And } \gamma_{v c}\left(K_{n}^{|t|}\right)=1 .
$$

Proof: Let $K_{n}^{|t|}$ be the t-tuple coalescence of complete graph $K_{n}$, the vertex connected dominating set of $K_{n}^{|t|}$ of size one is one and the vertex connected dominating set of $K_{n}^{|t|}$ of size $i-1$ is $t(n-1)$, then $d_{v c}\left(K_{n}^{|t|}, i\right)=\left(\begin{array}{c}t(n-1) \\ i-1\end{array}\right)$.

Theorem 2.12: The vertex connected domination polynomial of t-tuple coalescence of complete graph $K_{n}$ is given by

$$
D_{v c}\left(K_{n}^{|t|}, x\right)=x(x+1)^{t(n-1)} \text {, for all } \mathrm{n} \text { and } t \geq 2 .
$$

Proof: By Definition 1.1, we have:

$$
\begin{aligned}
D_{v c}\left(K_{n}^{|t|}, x\right) & =\sum_{i=1}^{t(n-1)+1} d_{v c}\left(K_{n}^{|t|}, i\right) x^{i}, \text { and by Theorem 2.11, we have } \\
D_{v c}\left(K_{n}^{|t|}, x\right) & =\sum_{i=1}^{t(n-1)+1}\left(\begin{array}{c}
t(n-1) \\
i-1
\end{array}\right) x^{i} \\
& =\left(\begin{array}{c}
t(n-1) \\
0
\end{array}\right) x+\left(\begin{array}{c}
t(n-1) \\
1
\end{array}\right) x^{2}+\left(\begin{array}{c}
t(n-1) \\
2
\end{array}\right) x^{3}+\ldots+\left(\begin{array}{c}
t(n-1) \\
t(n-1)
\end{array}\right) x^{t(n-1)+1} \\
& =x\left[\begin{array}{c}
1+\left(\begin{array}{c}
t(n-1) \\
1
\end{array}\right) x+\left(\begin{array}{c}
t(n-1) \\
2
\end{array}\right) x^{2}+\ldots+\left(\begin{array}{c}
t(n-1) \\
t(n-1)
\end{array}\right) x^{t(n-1)}
\end{array}\right] \\
& =x\left[\sum_{i=0}^{t(n-1)}\left(\begin{array}{c}
t(n-1) \\
i
\end{array}\right) x^{i}\right]=x(x+1)^{t(n-1)}
\end{aligned}
$$

Hence, $D_{v c}\left(K_{n}^{|t|}, x\right)=x(x+1)^{t(n-1)}$.

And the vertex connected dominating roots of $D_{v c}\left(K_{n}^{|t|}, x\right)$ are 0 with multiplicity 1 and -1 with multiplicity $t(n-1)$.

\section{Vertex Connected Domination Polynomial of wheel Coalescence Graphs}

In this section, we determine the vertex connected dominating set and vertex connected domination polynomial of wheel coalescence graphs. Also we determine the vertex connected dominating set and vertex connected domination polynomial of vertex identification and edge introducing between complete and wheel graphs.

Proposition 3.1: The following properties hold for the coefficients of $D_{v c}\left(W_{n_{1}} \bullet W_{n_{2}}, x\right)$, for all $\mathrm{n}_{1}, \mathrm{n}_{2} \geq 3$.

$$
\begin{array}{ll}
\text { i) } & d_{v c}\left(W_{n_{1}} \bullet W_{n_{2}}, 0\right)=0 . \\
\text { ii) } & d_{v c}\left(W_{n_{1}} \bullet W_{n_{2}}, 1\right)=d_{v c}\left(W_{n_{1}} \bullet W_{n_{2}}, n_{1}+n_{2}-1\right)=\left(\begin{array}{c}
n_{1}+n_{2}-2 \\
0
\end{array}\right)=1 . \\
\text { iii) } \quad & d_{v c}\left(W_{n_{1}} \bullet W_{n_{2}}, 2\right)=d_{v c}\left(W_{n_{1}} \bullet W_{n_{2}}, n_{1}+n_{2}-2\right)=\left(\begin{array}{c}
n_{1}+n_{2}-2 \\
1
\end{array}\right) . \\
\text { iv) } \quad & d_{v c}\left(W_{n_{1}} \bullet W_{n_{2}}, 3\right)=d_{v c}\left(W_{n_{1}} \bullet W_{n_{2}}, n_{1}+n_{2}-3\right)=\left(\begin{array}{c}
n_{1}+n_{2}-2 \\
2
\end{array}\right) .
\end{array}
$$




$$
\begin{aligned}
& \text { v) } \quad d_{v c}\left(W_{n_{1}} \bullet W_{n_{2}}, 4\right)=d_{v c}\left(W_{n_{1}} \bullet W_{n_{2}}, n_{1}+n_{2}-4\right)=\left(\begin{array}{c}
n_{1}+n_{2}-2 \\
3
\end{array}\right) . \\
& \text { vi) } \quad d_{v c}\left(W_{n_{1}} \bullet W_{n_{2}}, i\right)=d_{v c}\left(W_{n_{1}} \bullet W_{n_{2}}, n_{1}+n_{2}-i\right)=\left(\begin{array}{c}
n_{1}+n_{2}-2 \\
i-1
\end{array}\right)
\end{aligned}
$$

Theorem 3.2: The vertex connected dominating set of vertex identification of two wheel graphs is given by

$$
d_{v c}\left(W_{n_{1}} \bullet W_{n_{2}}, i\right)=\left(\begin{array}{c}
n_{1}+n_{2}-2 \\
i-1
\end{array}\right) .
$$

If $\mathrm{n}_{1}=\mathrm{n}_{2}=\mathrm{n}$, then $d_{v c}\left(W_{n} \bullet W_{n}, i\right)=\left(\begin{array}{c}2 n-2 \\ i-1\end{array}\right), \mathrm{i}=1,2, \ldots, 2 \mathrm{n}-1$.

Moreover, $\gamma_{v c}\left(W_{n_{1}} \bullet W_{n_{2}}\right)=1$.

Proof: By using Proposition 3.1, we get the result.

Theorem 3.3: The vertex connected domination polynomial of $W_{n_{1}} \bullet W_{n_{2}}$ is given by

$$
D_{v c}\left(W_{n_{1}} \bullet W_{n_{2}}, x\right)=x(x+1)^{n_{1}+n_{2}-2} .
$$

Proof: By using Definition 1.1, we have

$$
\begin{aligned}
& D_{v c}\left(W_{n_{1}} \bullet W_{n_{2}}, x\right)=\sum_{i=1}^{n_{1}+n_{2}-1} d_{v c}\left(W_{n_{1}} \bullet W_{n_{2}}, i\right) x^{i} \text {, and by Theorem 3.2, we have } \\
& D_{v c}\left(W_{n_{1}} \bullet W_{n_{2}}, x\right)=\sum_{i=1}^{n_{1}+n_{2}-1}\left(\begin{array}{c}
n_{1}+n_{2}-2 \\
i-1
\end{array}\right) x^{i} \\
& =\left(\begin{array}{c}
n_{1}+n_{2}-1 \\
0
\end{array}\right) x+\left(\begin{array}{c}
n_{1}+n_{2}-1 \\
1
\end{array}\right) x^{2}+\left(\begin{array}{c}
n_{1}+n_{2}-1 \\
2
\end{array}\right) x^{3}+\ldots+\left(\begin{array}{l}
n_{1}+n_{2}-1 \\
n_{1}+n_{2}-1
\end{array}\right) x^{n_{1}+n_{2}-1} \\
& =x\left[1+\left(\begin{array}{c}
n_{1}+n_{2}-1 \\
1
\end{array}\right) x+\left(\begin{array}{c}
n_{1}+n_{2}-1 \\
2
\end{array}\right) x^{2}+\ldots+\left(\begin{array}{l}
n_{1}+n_{2}-1 \\
n_{1}+n_{2}-1
\end{array}\right) x^{n_{1}+n_{2}-2}\right] \\
& =x\left[\sum_{i=0}^{n_{1}+n_{2}-2}\left(\begin{array}{c}
n_{1}+n_{2}-2 \\
i
\end{array}\right) x^{i}\right] \\
& =x(x+1)^{n_{1}+n_{2}-2} \text {. }
\end{aligned}
$$

Hence, $D_{v c}\left(W_{n_{1}} \bullet W_{n_{2}}, x\right)=x(x+1)^{n_{1}+n_{2}-2}$.

And the vertex connected dominating roots of $D_{v c}\left(W_{n_{1}} \bullet W_{n_{2}}, x\right)$ are 0 with multiplicity 1 and -1 with multiplicity $n_{1}+n_{2}-2$.

Proposition 3.4: The following properties hold for the coefficients of $D_{v c}\left(W_{n_{1}}: W_{n_{2}}, x\right)$, for all $n_{1}, n_{2} \geq 3$.

$$
\begin{aligned}
& \text { i) } d_{v c}\left(W_{n_{1}}: W_{n_{2}}, 0\right)=d_{v c}\left(W_{n_{1}}: W_{n_{2}}, 1\right)=0 . \\
& \text { ii) } d_{v c}\left(W_{n_{1}}: W_{n_{2}}, 2\right)=d_{v c}\left(W_{n_{1}}: W_{n_{2}}, n_{1}+n_{2}-2\right)=\left(\begin{array}{c}
n_{1}+n_{2}-2 \\
0
\end{array}\right)=1 . \\
& \text { iii) } d_{v c}\left(W_{n_{1}}: W_{n_{2}}, 3\right)=d_{v c}\left(W_{n_{1}}: W_{n_{2}}, n_{1}+n_{2}-3\right)=\left(\begin{array}{c}
n_{1}+n_{2}-2 \\
1
\end{array}\right) . \\
& \text { iv) } d_{v c}\left(W_{n_{1}}: W_{n_{2}}, 4\right)=d_{v c}\left(W_{n_{1}}: W_{n_{2}}, n_{1}+n_{2}-4\right)=\left(\begin{array}{c}
n_{1}+n_{2}-2 \\
2
\end{array}\right) . \\
& \text { v) } d_{v c}\left(W_{n_{1}}: W_{n_{2}}, i\right)=d_{v c}\left(W_{n_{1}}: W_{n_{2}}, n_{1}+n_{2}-i\right)=\left(\begin{array}{c}
n_{1}+n_{2}-2 \\
i-2
\end{array}\right)
\end{aligned}
$$

Theorem 3.5: The vertex connected dominating set of $W_{n_{1}}: W_{n_{2}}$ is given by 


$$
d_{v c}\left(W_{n_{1}}: W_{n_{2}}, i\right)=\left(\begin{array}{c}
n_{1}+n_{2}-2 \\
i-2
\end{array}\right)
$$

If $\mathrm{n}_{1}=\mathrm{n}_{2}=\mathrm{n}$, then $d_{v c}\left(W_{n}: W_{n}, i\right)=\left(\begin{array}{c}2 n-2 \\ i-2\end{array}\right), \mathrm{i}=2, \ldots, 2 \mathrm{n}$.

Moreover, $\gamma_{v c}\left(W_{n_{1}}: W_{n_{2}}\right)=2$.

Proof: By using Proposition 3.4, we get the result.

Theorem 3.6: The vertex connected domination polynomial of $W_{n_{1}}: W_{n_{2}}$ is given by

$$
D_{v c}\left(W_{n_{1}}: W_{n_{2}}, x\right)=x^{2}(x+1)^{n_{1}+n_{2}-2} .
$$

Proof: By Definition 1.1 and Theorem 3.5, we have

$$
\begin{aligned}
& D_{v c}\left(W_{n_{1}}: W_{n_{2}}, x\right)=\sum_{i=2}^{n_{1}+n_{2}} d_{v c}\left(W_{n_{1}}: W_{n_{2}}, i\right) x^{i}=\sum_{i=2}^{n_{1}+n_{2}}\left(\begin{array}{c}
n_{1}+n_{2}-2 \\
i-2
\end{array}\right) x^{i} \\
& =\left(\begin{array}{c}
n_{1}+n_{2}-2 \\
0
\end{array}\right) x^{2}+\left(\begin{array}{c}
n_{1}+n_{2}-2 \\
1
\end{array}\right) x^{3}+\left(\begin{array}{c}
n_{1}+n_{2}-2 \\
2
\end{array}\right) x^{4}+\ldots+\left(\begin{array}{l}
n_{1}+n_{2}-2 \\
n_{1}+n_{2}-2
\end{array}\right) x^{n_{1}+n_{2}} \\
& =x^{2}\left[1+\left(\begin{array}{c}
n_{1}+n_{2}-2 \\
1
\end{array}\right) x+\left(\begin{array}{c}
n_{1}+n_{2}-2 \\
2
\end{array}\right) x^{2}+\ldots+\left(\begin{array}{l}
n_{1}+n_{2}-2 \\
n_{1}+n_{2}-2
\end{array}\right) x^{n_{1}+n_{2}-2}\right] \\
& =x^{2}\left[\sum_{i=0}^{n_{1}+n_{2}-2}\left(\begin{array}{c}
n_{1}+n_{2}-2 \\
i
\end{array}\right) x^{i}\right] \\
& =x^{2}(x+1)^{n_{1}+n_{2}-2} \text {. }
\end{aligned}
$$

Hence, $D_{v c}\left(W_{n_{1}}: W_{n_{2}}, x\right)=x^{2}(x+1)^{n_{1}+n_{2}-2}$

And the vertex connected dominating roots of $D_{v c}\left(W_{n_{1}}: W_{n_{2}}, x\right)$ are 0 with multiplicity 2 and -1 with multiplicity $n_{1}+n_{2}-2$.

Theorem 3.7: The vertex connected dominating set of t-tuple coalescence of wheel graph $W_{n}$ is given by

$$
d_{v c}\left(W_{n}^{|t|}, i\right)=\left(\begin{array}{c}
t(n-1) \\
i-1
\end{array}\right) \text { and } \gamma_{v c}\left(W_{n}^{|t|}\right)=1
$$

Proof: The proof is similar to the proof of Theorem 2.11.

Theorem 3.8: The vertex connected domination polynomial of t-tuple coalescence of wheel graph $W_{n}$ is given by

$$
D_{v c}\left(W_{n}^{|t|}, x\right)=x(x+1)^{t(n-1)} .
$$

Proof: By using Definition 1.1 and Theorem 3.7, we get the result.

Theorem 3.9: The vertex connected dominating set and vertex connected domination polynomial of $K_{n_{1}} \bullet W_{n_{2}}$ is given by

$$
d_{v c}\left(K_{n_{1}} \bullet W_{n_{2}}, i\right)=\left(\begin{array}{c}
n_{1}+n_{2}-2 \\
i-1
\end{array}\right) \text { and } D_{v c}\left(K_{n_{1}} \bullet W_{n_{2}}, x\right)=x(x+1)^{n_{1}+n_{2}-2} .
$$

Proof: The proof is similar to the proof of Theorems 2.4 and 2.5.

Theorem 3.10: The vertex connected dominating set and vertex connected domination polynomial of $K_{n_{1}}: W_{n_{2}}$ is given by

$$
d_{v c}\left(K_{n_{1}}: W_{n_{2}}, i\right)=\left(\begin{array}{c}
n_{1}+n_{2}-2 \\
i-2
\end{array}\right) .
$$

If $n_{1}=n_{2}=n$, then $d_{v c}\left(K_{n}: W_{n}, i\right)=\left(\begin{array}{c}2 n-2 \\ i-2\end{array}\right), \mathrm{i}=1,2, \ldots, 2 \mathrm{n}-1$.

$$
\text { Moreover, } \gamma_{v c}\left(K_{n_{1}}: W_{n_{2}}\right)=2 \text { and } D_{v c}\left(K_{n_{1}}: W_{n_{2}}, x\right)=x^{2}(x+1)^{n_{1}+n_{2}-2} \text {. }
$$

Proof: The proof is similar to the proof of the Theorems 2.9 and 2.10. 


\section{Conclusion}

In this paper, we studied the vertex connected domination polynomials of some graph coalescence. In the future one can compute the vertex domination polynomial of some other coalescence and t-tuple of other graphs.

\section{References}

[1] A. Vijayan, T.B. Anitha, G. Edwin, Connected Total Dominating Sets and Connected Total Domination Polynomials of Stars and Wheels, IOSR Journal of Mathematics, Vol. 11, No. 1, pp. 112-121, 2015.

[2] B. Askari and M. Alaeiyan, The vertex domination polynomial and edge domination polynomial of a graph, Acta Universitatis Apulensis, Vol. 28, pp. 157-162, 2011.

[3] B. Chaluvaraju , Puttaswamy, N. Manjunath and S. R. Nayaka, The co-connected Domination Polynomial of a Graph, Advances and Applications in Discrete Mathematics, Vol. 18, No. 1, pp.57-70, 2017.

[4] C. E. Go and S. R. Canoy, Jr., Domination in the corona and join of graphs, Int. Math. Forum, Vol. 6, No.16, pp. 763-771, 2011.

[5] E. Sampathkumar, H. B. Walikar, The Connected Domination Number of a Graph, J. Math. Phys. Sci., Vol.13, No. 6, pp. 607$613,1979$.

[6] F. Harary, Graph theory, Addison-Wesley, Reading Mass, 1969.

[7] J. Brown and J. Tufts, On the roots of domination polynomials, Graphs and Combinatorics, 2013.

[8] K. R. Sharaf, D. A. Ali, Nullity of t-Tupple Graphs, International Journal of Mathematical, Computational, Statistical, Natural and Physical Engineering Vol. 8, No. 2, pp. 314-324, 2014.

[9] M. B. V. Dhananjaya, G. Deepak, N. D. Soner, Connected Domination Polynomial of a Graph, International Journal of Mathematical Archive- Vol. 4 No. 11, pp. 90-96, 2013.

[10] N. B. Ibrahim1 and H. J. Ahmed, Results on the Domination Polynomial of Some Coalescence Graphs, Journal of Zankoy Sulaimani, Vol. 19, No. 1, pp.171-176, 2017.

[11] S. Alikhani and Y.-H. Peng, Dominating sets and domination polynomials of certain graphs. II, Opuscula Mathematica, Vol. 30 , No. 1, pp. 37-51, 2010.

[12] S. Alikhani, Dominating sets and domination polynomials of graphs, Ph.D. thesis, Universiti Putra Malaysia, 2009.

[13] S. Alikhani, Y-h. Peng, Introduction to Domination Polynomial of a Graph, arXiv: 0905.2251v1, math.CO, 2009.

[14] T. Kotek, J. Preen, Recurrence relations and splitting formulas for the domination polynomial, The .Electronic Journal of Combinatorics, Vol. 19, No. 3, pp. 1-27, 2012.

[15] T. W. Haynes, S. T. Hedetniemi and P. J. Slater, Fundamentals of Domination in Graphs, Marcel Dekker, Inc., New York, 1998.

\section{Authors' Profiles}

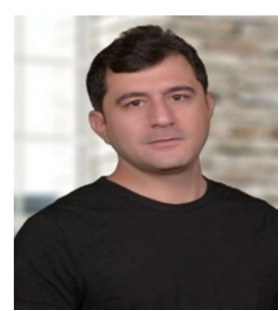

Nechirvan Badal Ibrahim is currently Lecturer and Ph.D student in the Department of Mathematics Duhok University, Kurdistan Region Iraq. His research areas are Graph Theory and Topological Graph Theory. He is a coordinator of Department of Mathematics Duhok University.

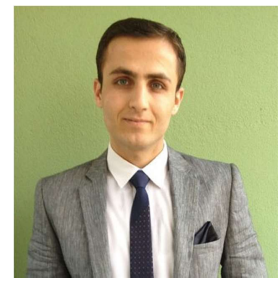

Hariwan Fadhil M.Salih is currently Lecturer in the Department of Mathematics Duhok University, Kurdistan Region Iraq. His research areas are Number Theory and Applied Mathematics.

How to cite this paper: Nechirvan Badal Ibrahim, Hariwan Fadhil M.Salih. " Vertex Connected Domination Polynomial of some Coalescence of Complete and Wheel Graphs ", International Journal of Mathematical Sciences and Computing (IJMSC), Vol.6, No.6, pp.1-8, 2020. DOI: 10.5815/IJMSC.2020.06.01 